\title{
Differential expression patterns of the short and long isoform of cFLIP on FasL-mediated apoptosis
}

\author{
HEE JUNG UM, ANIL KUMAR CHAUHAN, KYOUNG-JIN MIN and TAEG KYU KWON \\ Department of Immunology, School of Medicine, Keimyung University, Dalseo-gu, Daegu 704-701, Republic of Korea
}

Received November 6, 2017; Accepted March 12, 2018

DOI: $10.3892 /$ or.2018.6317

\begin{abstract}
FLIP is a key regulator of the anti-apoptotic mechanism and its association with FAS-mediated apoptosis has been widely studied and well documented. However, the equipoise between its two isoforms i.e. the long isoform cFLIP(L) and the short isoform cFLIP(S) during FAS-mediated apoptosis remains to be revealed. Therefore, the present study aimed to investigate the regulatory effect of these isoforms on FasL-mediated apoptosis in renal carcinoma. Our results revealed that FasL treatment to Caki cells induced the expression of cFLIP(S) and downregulated the expression of cFLIP(L) in a concentration- and time-dependent manner. Furthermore, our results indicated that cell death receptor-mediated apoptosis inducers such as TNF- $\alpha$ and TRAIL, induced apoptosis in Caki cells along with downregulation of cFLIP(L), however, they had no effect on the expression of cFLIP(S). In addition, FasL-mediated cFLIP(L) downregulation was prevented by pan-caspase inhibitor (z-VAD-fmk), however pan-caspase inhibitor did not have an effect on FasL-induced cFLIP(S) upregulation. Furthermore, FasL induced upregulation of the expression of $\mathrm{cFLIP}(\mathrm{S})$ at the post-translational level. Furthermore, pretreatment of Caki cells with ROS scavengers (N-acetylcysteine and glutathione) prevented the downregulation of cFLIP(L), the upregulation of $\mathrm{cFLIP}(\mathrm{S})$ and apoptosis induced by FasL. Collectively, these data indicated that a novel pathway of cFLIP(L)/(S) differential expression pattern was associated with FasL-induced apoptosis and modulated by ROS generation.
\end{abstract}

Correspondence to: Professor Taeg Kyu Kwon, Department of Immunology, School of Medicine, Keimyung University, 2800 Dalgubeoldaero, Dalseo-gu, Daegu 704-701, Republic of Korea E-mail:kwontk@dsmc.or.kr

Abbreviations: TRAIL, tumor necrosis factor-related apoptosisinducing ligand; ROS, reactive oxygen species; cFLIP, cellular FLICE-like inhibitory protein; TNF, tumor necrosis factor

Key words: FasL, cFLIP(L)/(S), apoptosis, ROS

\section{Introduction}

Apoptosis is defined as a programmed cell death which is responsible for the maintenance of tissue homeostasis during normal physiological as well as pathological conditions. There are a variety of cell surface receptors that contribute to the regulation of the apoptosis mechanism, for example, surface immunoglobulin, T-cell antigen receptor, and tumor necrosis receptor (1-3). Among these surface receptors, Fas belongs to the tumor necrosis factor (TNF) receptor family of death receptors and is able to initiate the apoptosis pathway through binding to specific death ligands of the cells which include, Fas ligand (FasL), tumor necrosis factor- $\alpha$ (TNF- $\alpha$ ) and Fas-specific monoclonal antibody CH11 (mAb CH11) $(4,5)$. Fas-mediated apoptosis is believed to play a crucial role in cytotoxic T-cell and natural killer cell-mediated apoptosis in cancer, as their activation leads to the expression of Fas ligand on the cell surface which eventually induces apoptosis in targeted cancer cells (6-8).

The induction of apoptosis by Fas is sophisticated and it starts with the formation of the death-inducing signaling complex (DISC) which acts as the cellular switch for the apoptosis pathway. However, the DISC formation initiates right after the interaction of FasL to Fas, followed by the aggregation of receptor molecules and the recruitment of the Fas-associated death domain (FADD), through the death domain interaction. Furthermore FADD contains a death effector domain that leads to the recruitment of pro-caspase- 8 resulting in the formation of a multimeric protein complex and the activation of caspase-8 $(9,10)$.

Conversely, there are several proteins that have a regulatory effect on Fas-mediated apoptosis, which when activated, suppress the apoptosis pathway induced by Fas. Among these, a key protein that regulates Fas-mediated apoptosis at DISC level, is cellular FLICE-like inhibitory protein (cFLIP). cFLIP interferes with caspase to prevent the cleavage and activation of caspase (11). cFLIP has been extensively studied for its anti-apoptotic potential and is found to be expressed in various cancer cells, for example, ovarian, colon, glioblastoma, breast, colorectal, renal and prostate cancer in a considerable amount where it is found to cause TRAIL resistance (12-14). Furthermore, cFLIP has been reported having 11 distinct splicing variants, three among which are expressed predominantly i.e. cFLIP(L), cFLIP(S) and cFLIP(R) $(12,15)$. Apart from the other two variants, cFLIP(L) has gained attention in 
the scientific community as a major variant involved in the blockage of caspase-8/10 activation. However, comprehensive studies on cFLIP(L) revealed that it had binary function, for example, ectopic overexpression of cFLIP(L) inhibited the activation of caspase- 8 and ectopic expression equivalent to endogenous cFLIP(L) was found to promote caspase- 8 processing in HeLa and MCF cells $(16,17)$. Therefore, the role of cFLIP(L) soon became controversial. Furthermore, despite the fact that $\mathrm{cFLIP}(\mathrm{S})$ also plays an important role in Fas-mediated apoptosis, cFLIP(S) remains neglected, with few exceptions $(18,19)$. These observations were the motivation behind the present study.

In the present study we examined the regulation of both isoforms of cFLIP (long and short) during Fas-mediated apoptosis in renal carcinoma cell lines and revealed that both isoforms are differentially regulated during Fas-mediated apoptosis at the translational level, however this expression was unaffected at the transcriptional level.

\section{Materials and methods}

Cells and materials. Caki-1 cells were obtained from the American Type Culture Collection (ATCC, Manassas, VA, USA). The culture medium used throughout these experiments was Dulbecco's modified Eagle's medium (DMEM; WelGene, Inc., Daegu, Korea), containing $10 \%$ fetal bovine serum (FBS; WelGene), $20 \mathrm{mM}$ HEPES buffer and $100 \mathrm{mg} / \mathrm{ml}$ gentamycin. Anti-Fas antibody (human, activating) clone $\mathrm{CH} 11$ $(1: 5,000 ; 1: 2,500 ; 1: 1,000$; cat. no. 05-201) was purchased from EDM Millipore (Darmstadt, Germany). Anti-caspase-3 (1:700; cat. no. ADI-AAP-113) and anti-c-FLIP (1:700; cat. no. ALX-804-961-0100) antibodies were purchased from Enzo Life Sciences (Farmington, NY, USA). Anti-PARP antibody (1:700; cat. no. 9542S) was purchased from Cell Signaling Technology (Beverly, MA, USA). Other chemicals were obtained from Sigma-Aldrich (St. Louis, MO, USA).

Western blotting. The cells were washed with cold PBS and lysed on ice in $50 \mu \mathrm{l}$ of lysis buffer $(50 \mathrm{mM}$ Tris- $\mathrm{HCl}$, $1 \mathrm{mM}$ EGTA, 1\% Triton X-100, $1 \mathrm{mM}$ phenylmethylsulfonyl fluoride, $\mathrm{pH} 7.5)(20,21)$. Lysates were centrifuged at $10,000 \mathrm{x} \mathrm{g}$ for $15 \mathrm{~min}$ at $4^{\circ} \mathrm{C}$ and the supernatant fractions were collected. Proteins were separated by SDS-PAGE and transferred to an Immobilon-P membrane (GE Healthcare Life Science, Marlborough, MA USA). Specific proteins were detected using an enhanced chemiluminescence (ECL) western blot kit (EMD Millipore) according to the manufacturer's instructions.

Cell count and flow cytometric analysis. Cell counts were performed using a hemocytometer (Marienfeld-Superior, Lauda-Königshofen, Germany). Approximately $0.4 \times 0^{6}$ Caki cells were suspended in $100 \mathrm{ml}$ of PBS and $200 \mathrm{ml}$ of $95 \%$ ethanol were added while vortexing. The cells were incubated at $4^{\circ} \mathrm{C}$ for $1 \mathrm{~h}$, washed with PBS and resuspended in $250 \mathrm{ml}$ of $1.12 \%$ sodium citrate buffer ( $\mathrm{pH} \mathrm{8.4)} \mathrm{together}$ with $12.5 \mathrm{mg}$ of RNase. Incubation continued at $37^{\circ} \mathrm{C}$ for $30 \mathrm{~min}$. The cellular DNA was then stained by applying $250 \mathrm{ml}$ of propidium iodide (PI; $50 \mathrm{mg} / \mathrm{ml}$ ) for $30 \mathrm{~min}$ at room temperature. The stained cells were analyzed by fluorescent activated cell sorting (FACS) on a FACScan flow cytometer
(BD Biosciences, San Jose, CA, USA) for relative DNA content based on red fluorescence.

RNA isolation and RT-PCR. To determine whether the potential sensitizing effects of FasL-mediated apoptosis were a result of increased levels of mRNA encoding cFLIP(L) and cFLIP(S), we compared the levels of cFLIP in Caki cells, which were treated with or without various concentrations of FasL. cFLIP mRNA expression was determined by RT-PCR. Total cellular RNA was extracted from cells using the TRIzol reagent (Life Technologies; Thermo Fisher Scientific, Inc., Waltham, MA, USA). A cDNA was synthesized from $2 \mu \mathrm{g}$ of total RNA using M-MLV reverse transcriptase (Gibco-BRL; Thermo Fisher Scientific). The cDNA for cFLIP(L) and cFLIP(S) was amplified by PCR with specific primers. The primer sequences for $\mathrm{cFLIP}(\mathrm{L})$ and $\mathrm{cFLIP}(\mathrm{S})$ were as follows: Sense for cFLIP(L) and cFLIP(S), 5'-CGGACTATAGAGTGC TGATGG-3' and antisense for cFLIP(L), 5'-GATTATCAG GCAGATTCCTAG-3' and for cFLIP(S), 5'-AGATCAGGA CAATGGGCATAG-3'.

Small-interfering RNAs (siRNAs). The GFP (control) siRNA duplexes used in the present study were purchased from Santa Cruz Biotechnology (Santa Cruz, CA, USA). cFLIP(S) and cFLIP(L) siRNA duplexes were obtained from Invitrogen (Thermo Fisher Scientific). The sequences of cFLIP(S) and cFLIP(L) were AACAUGGAACUGCCUCUACUU and AAG GAACAGCUUGGCGCUCAA, respectively. The cells were transfected with siRNA oligonucleotides using Oligofectamine reagent (Invitrogen; Thermo Fisher Scientific) according to the manufacturer's instructions.

Statistical analysis. The data were analyzed using a one-way ANOVA and post hoc comparisons (Student-Newman-Keuls) using the Statistical Package for Social Sciences 22.0 software (SPSS, Inc., Chicago, IL, USA). P $<0.05$ were considered to indicate a statistically significant result.

\section{Results}

Effect of FasL treatment on the expression levels of cFLIP $(L)$ and $c F L I P(S)$ in human renal cancer cells. To determine the expression pattern of cFLIP(L) and cFLIP(S) during FasL-mediated apoptosis, the renal carcinoma Caki cells were treated with various concentrations of FasL (100-500 ng/ml) and then, flow cytometric and western blot analyses were performed to evaluate the apoptosis induction and the expression pattern of cFLIP(L) and cFLIP(S). FasL treatment caused an increase in sub-G1 population in a concentration- and time-dependent manner (Fig. 1A and D) which was further verified by the PARP cleavage in western blot analysis (Fig. 1B and E). The expression of cFLIP(L) was found to be highly downregulated after treatment of FasL. However, the expression of $\mathrm{cFLIP}(\mathrm{S})$ was significantly upregulated in a concentration- and time-dependent manner (Fig. 1B and E). In addition, there were no changes in either of the cFLIP variants at the mRNA levels (Fig. $1 \mathrm{C}$ and F), indicating that FasL treatment induced differential expression patterns of cFLIP(L) and cFLIP(S) and this expression was regulated at the post-transcriptional level in Caki cells. 
A

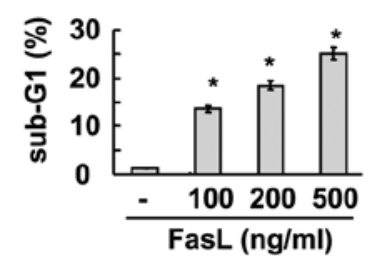

B

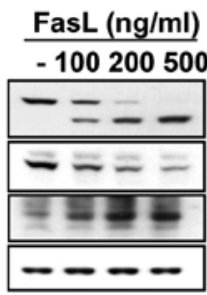

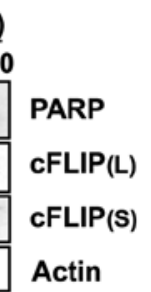

C

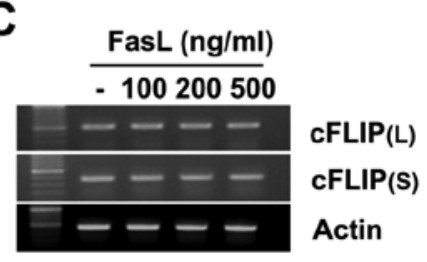

D

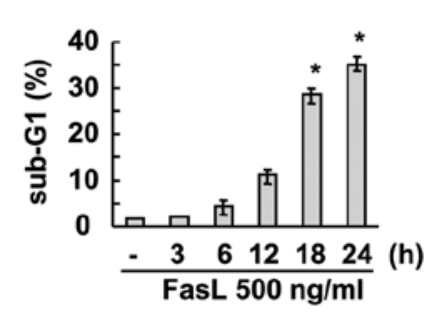

E

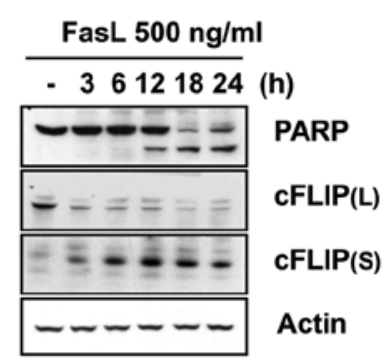

F

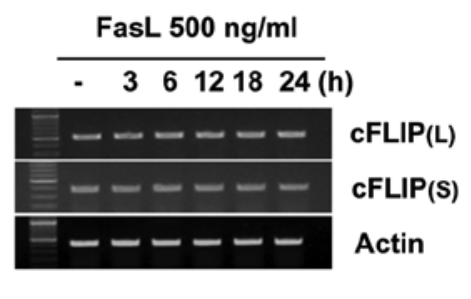

G

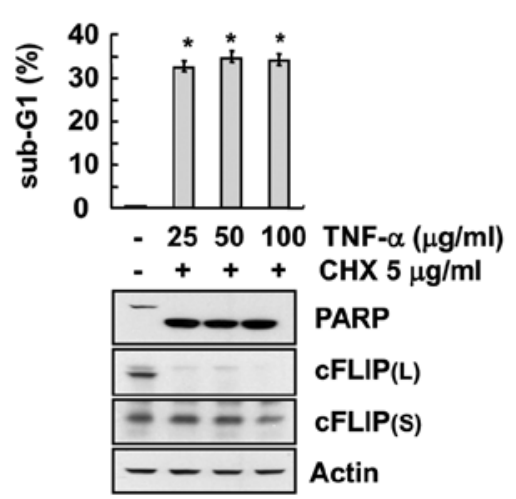

H

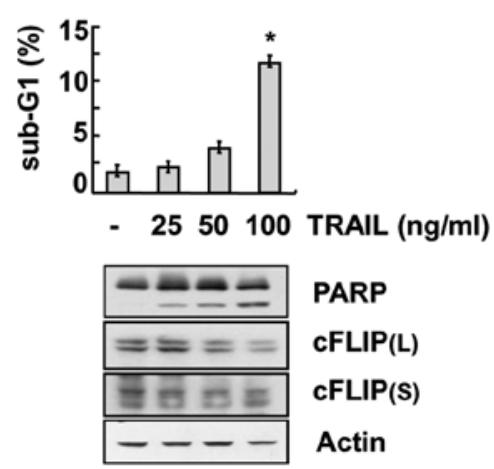

Figure 1. FasL induces downregulation of cFLIP(L) and upregulation of cFLIP(S) in human renal cancer cells. (A-C) Caki cells were treated with the indicated concentrations of FasL for $24 \mathrm{~h}$. The level of apoptosis was assessed by the sub-G1 fraction using flow cytometry (A). The protein expression levels of PARP, cFLIP(L), cFLIP(S) and actin were determined by western blotting (B). The mRNA expression levels of cFLIP(L), cFLIP(S) and actin were determined by RT-PCR (C). (D-F) Caki cells were treated with $500 \mathrm{ng} / \mathrm{ml} \mathrm{FasL} \mathrm{for} \mathrm{the} \mathrm{indicated} \mathrm{time-points.} \mathrm{The} \mathrm{level} \mathrm{of} \mathrm{apoptosis} \mathrm{was} \mathrm{determined} \mathrm{by} \mathrm{the} \mathrm{sub-G1} \mathrm{fraction}$ using flow cytometry (D). The protein expression levels of PARP, cFLIP(L), cFLIP(S) and actin were determined by western blotting (E). The mRNA expression levels of cFLIP(L), cFLIP(S) and actin were determined by RT-PCR (F). (G-H) Caki cells were treated with the indicated concentrations of TNF- $\alpha$ plus $5 \mu \mathrm{g} / \mathrm{ml}$ cycloheximide $(\mathrm{CHX})(\mathrm{G})$ or TRAIL $(\mathrm{H})$ for $24 \mathrm{~h}$. The level of apoptosis was determined by the sub-G1 fraction using flow cytometry. The protein expression levels of PARP, cFLIP(L), cFLIP(S) and actin were determined by western blotting. The values in A, D, G and $H$ represent the means \pm SD from three independent samples. ${ }^{*} \mathrm{P}<0.01$ compared to the control.

Furthermore, we investigated the effect of other cell death-receptor mediated apoptosis inducers (TNF- $\alpha$ and TRAIL) on the expression pattern of cFLIP variants with the treatment of various concentrations of TNF- $\alpha$ or TRAIL to Caki cells. The results of the experiment indicated that both TNF- $\alpha$ and TRAIL induced apoptosis in Caki cells along with downregulation of cFLIP(L) (Fig. $1 \mathrm{G}$ and $\mathrm{H}$ ). However, they had no effect on the expression of cFLIP(S) (Fig. 1G and H).

Role of caspase in FasL-mediated differential expression of $c F L I P(L)$ and $c F L I P(S)$ in human renal cancer cells. Based on the above mentioned data we observed that FasL-mediated apoptosis differentially regulated the expression of cFLIP(L) and cFLIP(S). Therefore, we determined whether this regulation was dependent on caspase regulation. In order to examine the role of caspase in FasL-mediated differential expression of cFLIP variants, treatment with pan-caspase inhibitor (z-VAD-fmk) was applied which inhibited FasL-mediated apoptosis (Fig. 2A). Inhibition of caspase prevented the downregulation of cFLIP(L). However, z-VAD did not have an effect on FasL-induced cFLIP(S) upregulation and mRNA levels of cFLIP(L) and cFLIP(S) (Fig. 2B and C). We examined whether both cFLIP variants play a role in FasL-induced apoptosis. Downregulation of each cFLIP variant by specific siRNA induced increased cell apoptotic populations in Fas L-treated Caki cells, compared with the control siRNA (Fig. 2D and E).

Effect of proteasome inhibitors and MAPK inhibitors on the expression of cFLIP $(L) /(S)$ in FasL-treated cells. RT-PCR analysis revealed that the mRNA levels of $\operatorname{cFLIP}(\mathrm{L}) /(\mathrm{S})$ were unchanged following FasL treatment. To investigate the possible involvement of the proteasome pathway in the FasL-mediated regulation of $\operatorname{cFLIP}(\mathrm{L}) /(\mathrm{S})$, we assessed the expression levels of $\mathrm{cFLIP}(\mathrm{L}) /(\mathrm{S})$ in cells treated with various proteasome inhibitors $(0.5 \mathrm{mM}$ MG132, $5 \mathrm{mM}$ ALLN and $5 \mathrm{mM}$ lactacystin) in the presence or absence of FasL. As displayed in Fig. 3A, FasL-induced cFLIP(S) upregulation and cFLIP(L) downregulation was not inhibited by any of the proteasome-inhibitors treatment. These results 
A

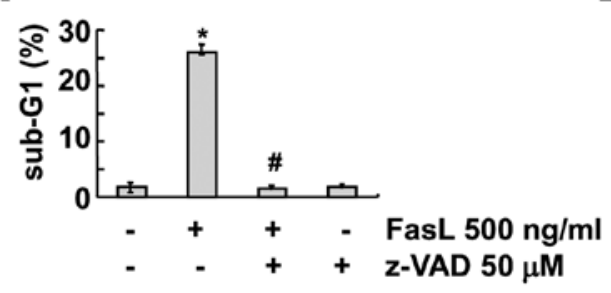

B

$-++-F a s L 500 \mathrm{ng} / \mathrm{ml}$ - $+\quad+\quad$ z-VAD $50 \mu \mathrm{M}$

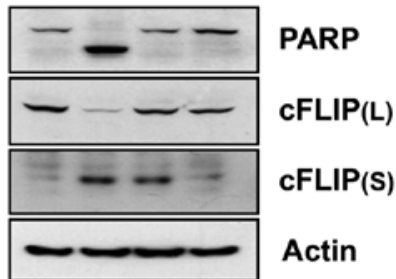

C

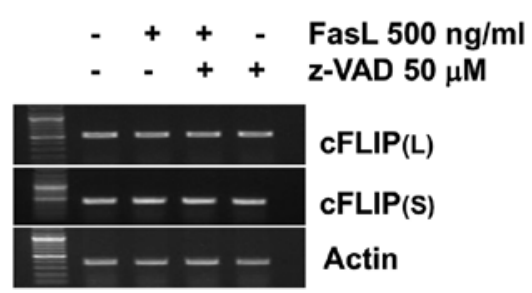

D

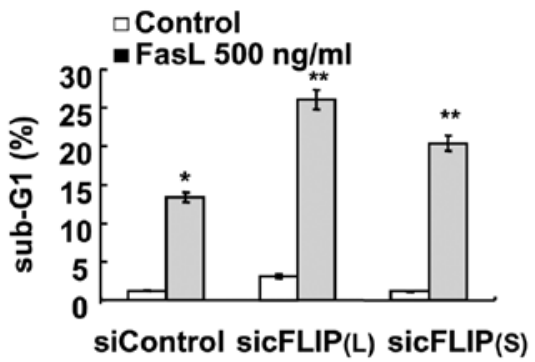

E

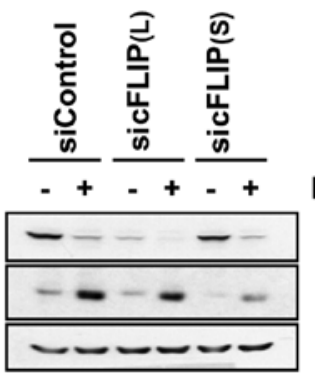

FasL $500 \mathrm{ng} / \mathrm{ml}$

cFLIP(L)

cFLIP(S)

Actin

Figure 2. Caspase activation does not affect the upregulation of the expression of FasL-induced cFLIP(S). (A-C) Caki cells were pretreated with 50 $\mu \mathrm{M} z-\mathrm{VAD}$ for $30 \mathrm{~min}$, and then $500 \mathrm{ng} / \mathrm{ml}$ FasL was added for $24 \mathrm{~h}$. The level of apoptosis was determined by the sub-G1 fraction using flow cytometry (A). The protein expression levels of PARP, cFLIP(L), cFLIP(S) and actin were determined by western blotting (B). The mRNA expression levels of cFLIP(L), cFLIP(S) and actin were determined by RT-PCR (C). (D-E) Caki cells were transiently transfected with control, cFLIP(L) or cFLIP(S) siRNA. After transfection, Caki cells were treated with $500 \mathrm{ng} / \mathrm{ml}$ FasL for $24 \mathrm{~h}$. The level of apoptosis was determined by the sub-G1 fraction using flow cytometry (D). The protein expression levels of PARP, cFLIP(L), cFLIP(S) and actin were determined by western blotting (E). The values in A and D represent the means \pm SD from three independent samples. ${ }^{*} \mathrm{P}<0.01$ compared to the control. ${ }^{*} \mathrm{P}<0.01$ compared to FasL. ${ }^{* *} \mathrm{P}<0.05$ compared to FasL-treated siControl.

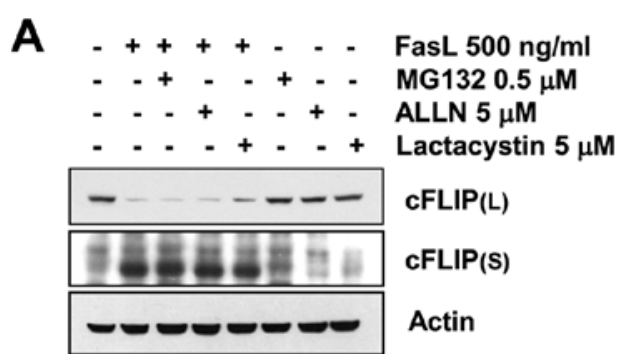

C

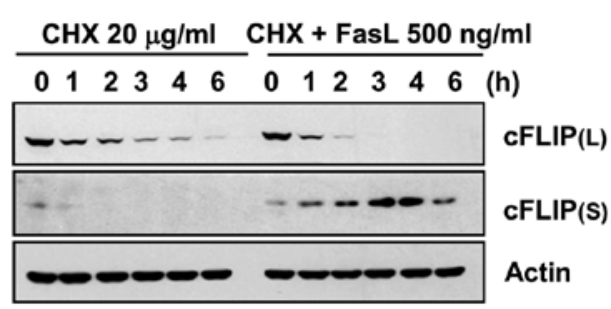

B
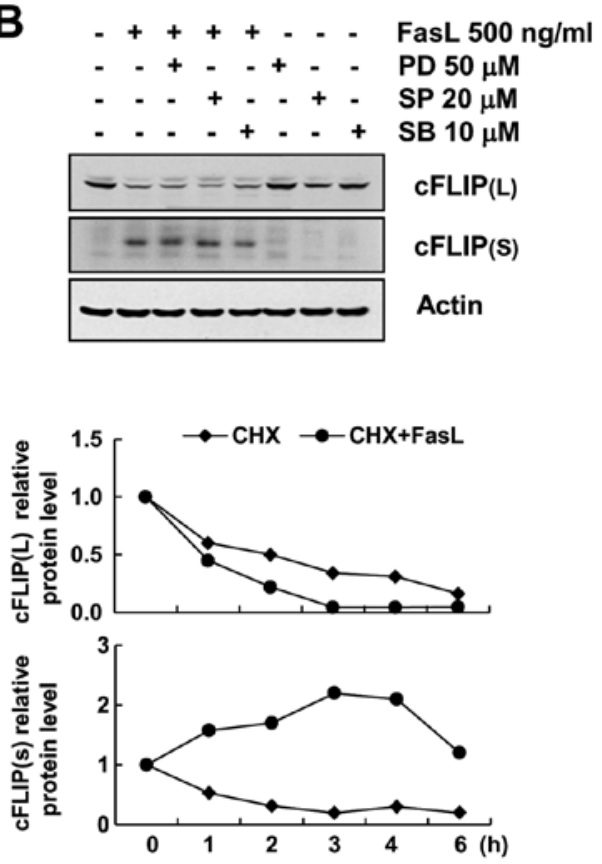

Figure 3. FasL induces upregulation of cFLIP(S) expression at the post-translational level. (A) Caki cells were pretreated with $0.5 \mu \mathrm{M}$ MG132, $5 \mu \mathrm{M}$ ALLN and $5 \mu \mathrm{M}$ lactacystin for $30 \mathrm{~min}$ and then treated with $500 \mathrm{ng} / \mathrm{ml} \mathrm{FasL}$ for $24 \mathrm{~h}$ (B) Caki cells pretreated with $50 \mu \mathrm{M}$ PD98059 (PD), $20 \mu \mathrm{M} \mathrm{SP600125} \mathrm{(SP)}$ and $10 \mu \mathrm{M} \mathrm{SB} 203580$ (SB) for $30 \mathrm{~min}$ and then treated with $500 \mathrm{ng} / \mathrm{ml} \mathrm{FasL}$ for $24 \mathrm{~h}$. (C) Caki cells were treated with $20 \mu \mathrm{g} / \mathrm{ml}$ cycloheximide (CHX) in the presence or absence of $500 \mathrm{ng} / \mathrm{ml}$ FasL for the indicated time-points. The protein expression levels of PARP, cFLIP(L), cFLIP(S) and actin were determined by western blotting. 

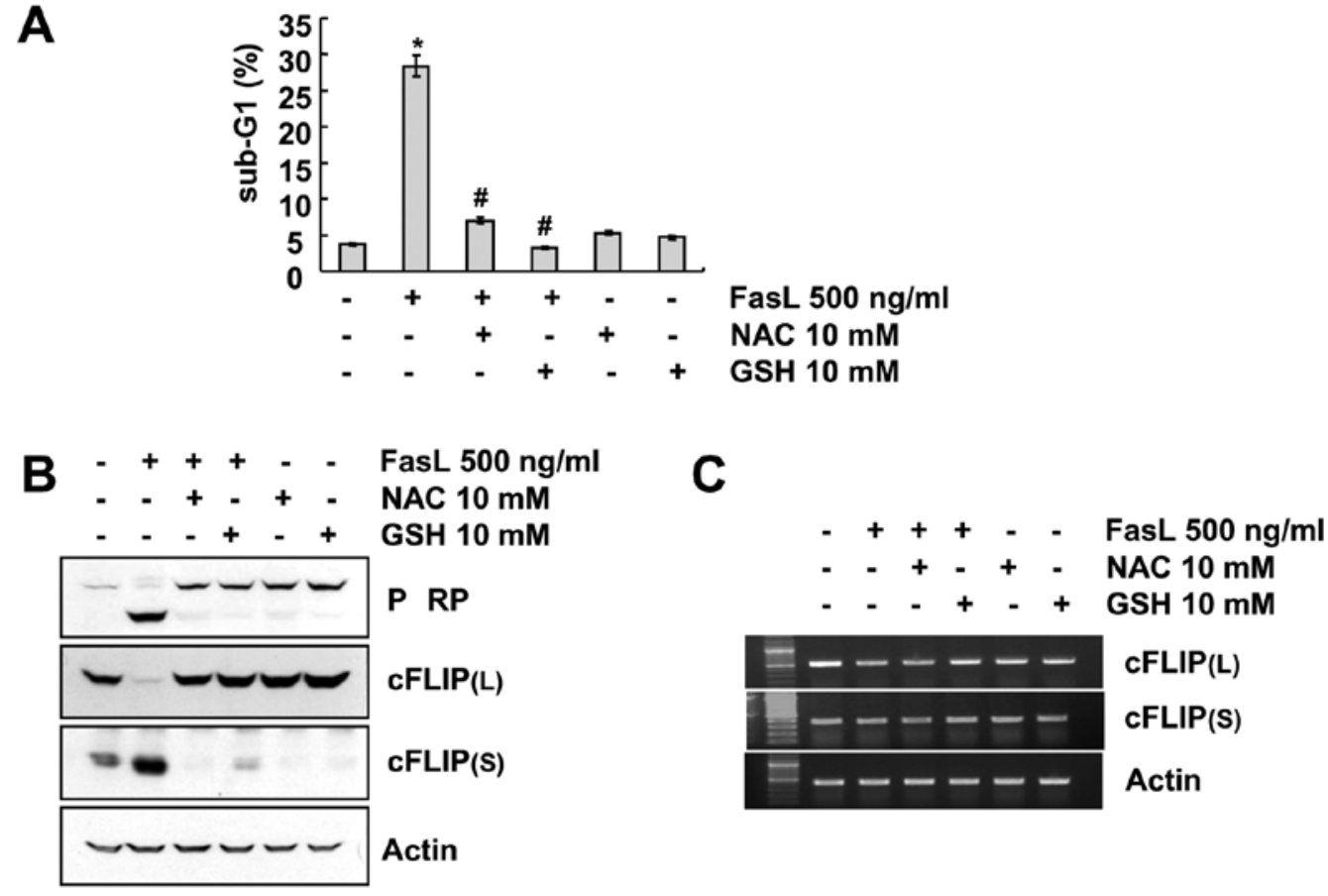

Figure 4. ROS contributes to FasL-induced upregulation of the expression of cFLIP(S). Caki cells were pretreated with 10 mM N-acetylcysteine (NAC) and $10 \mathrm{mM}$ glutathione (GSH) for $30 \mathrm{~min}$ and then treated with $500 \mathrm{ng} / \mathrm{ml} \mathrm{FasL}$ for $24 \mathrm{~h}$. The level of apoptosis was determined by the sub-G1 fraction using flow cytometry (A). The protein expression levels of PARP, cFLIP(L), cFLIP(S) and actin were determined by western blotting (B). The mRNA expression levels of cFLIP(L), cFLIP(S) and actin were determined by RT-PCR (C). The values in A represent the means \pm SD from three independent samples. ${ }^{*} \mathrm{P}<0.01$ compared to control. " $\mathrm{P}<0.01$ compared to FasL. ROS, reactive oxygen species.

indicated that proteasomal degradation was not associated with FasL-induced cFLIP expression regulation. In addition, in order to investigate whether the MAPKs pathways were involved in FasL-treated Caki cells, Caki cells were treated with each MAPK specific inhibitor. As displayed in Fig. 3B, FasL-induced cFLIP(S) upregulation and cFLIP(L) downregulation was not inhibited by MAPKs inhibitors treatment. These results indicated that MAPK signaling pathway was not associated with FasL-induced cFLIP expression regulation.

Not with standing, we examined whether protein stability was directly associated with FasL-induced expression regulation of $\mathrm{cFLIP}(\mathrm{L}) /(\mathrm{S})$. In order to investigate this, we treated Caki cells with cycloheximide (CHX), an inhibitor of protein translation. Untreated and FasL-treated Caki cells were exposed to $\mathrm{CHX}$ for various time-points and $\mathrm{cFLIP}(\mathrm{L})$ and cFLIP(S) protein levels were determined by western blot analysis. As displayed in Fig. 3C, FasL treatment caused much more rapid cFLIP(L) degradation than that of untreated cells. Notably, cFLIP(S) protein levels were maintained until $6 \mathrm{~h}$, and then declined in the presence of FasL and CHX. However, cFLIP(S) protein levels rapidly degraded by CHX treatment alone. Therefore, these results demonstrated that FasL-induced cFLIP(S) upregulation was associated with enhanced stability of cFLIP(S) protein.

FasL-mediated apoptosis and expression regulation of cFLIP are caused by ROS generation. Recently, Fas death signaling pathway has been reported to have an association with reactive oxygen species (ROS) generation and cFLIP(L) downregulation (22). Therefore, we examined whether ROS generation could be involved in FasL-mediated differential expression pattern of cFLIP(L)/(S) in Caki cells along with its direct association with FasL-induced apoptosis. As displayed in Fig. 4, pretreatment with ROS scavengers, $\mathrm{N}$-acetylcysteine (NAC) and glutathione (GSH), markedly blocked FasL-induced apoptosis and attenuated the cleavage of PARP (Fig. 4A and B). In addition, FasL-mediated cFLIP(L) downregulation and cFLIP(S) upregulation was prevented by NAC and GSH treatment (Fig. 4B). The RT-PCR data revealed that cFLIP(L)/(S) protein expression levels were not controlled by the transcriptional regulation, as there was no change in $\mathrm{cFLIP}(\mathrm{L}) /(\mathrm{S})$ mRNA level regardless of whether the cells were treated with FasL in the presence or absence of ROS scavengers (Fig. 4C). Collectivelly, these data clearly indicated that FasL-induced apoptosis and expression regulation of cFLIP were mediated by ROS generation.

\section{Discussion}

In the present study, we explored the differential regulation patterns of two major variants of cFLIP i.e. cFLIP(L) and cFLIP(S) during Fas-mediated apoptosis and found that the short form of cFLIP was upregulated. In addition, this differential regulation pattern of cFLIP(S) was observed at a post-translational level but not at a transcriptional level. In addition, our data demonstrated that the upregulation of cFLIP(S) was associated with ROS generation.

cFLIP is a well-recognized anti-apoptotic protein and it has been reported that both the principle variants of this protein, cFLIP(L) and cFLIP(S), are able to bind to the death effector domain (DED) region of the adaptor protein FADD along with caspase-8 and 10 (16). However, structurally, cFLIP(S) 
contains two tandem DEDs and cFLIP(L) contains only one tandem, thus, cFLIP(S) is believed to have a more potent role in the prevention of caspase activation than $\operatorname{cFLIP}(\mathrm{L})(23,24)$. Although some studies have been carried out to elucidate the role of these variants during death receptor-mediated apoptosis, the expression pattern of both variants and the principle behind their expression remains largely unclear.

In order to explore this, we have treated Caki cells with FasL and evaluated the expression pattern of cFLIP(S) and cFLIP(L). We found that FasL treatment caused the downregulation of cFLIP(L) and the upregulation of cFLIP(S) which was concentration- and time-dependent. Furthermore, the differential expression of both variants was observed at a post-translational but not at a transcriptional level. However, as above stated, these variants were able to prevent caspase activation. Therefore we examined their expression level by using pan-caspase inhibitor (z-VAD). Notably, inhibition of caspase prevented the downregulation of $\operatorname{cFLIP}(\mathrm{L})$, however, it did not have an effect on FasL-induced cFLIP(S) upregulation. We observed that knockdown of any cFLIP variant induced apoptosis, indicating that both variants were involved in the protection of FasL-mediated apoptosis in Caki cells. However, a study carried out by Chang et al (17), also revealed a similar expression pattern of cFLIP(S) in MCF cells.

Although, numerous studies have been carried out on Fas-mediated apoptosis which revealed its molecular mechanism from activation to apoptosis, more recent studies have reported the role of ROS in the activation of FasL-mediated apoptosis $(25,26)$. Excessive generation of free radicals could cause the damage of plasma membrane integrity resulting in leakage of cellular material which eventually leads to cell death (25). Notwithstanding, excessive ROS production has been reported to play a role in the activation of caspase (27) and the downregulation the cFLIP proteins (22). Therefore, in the present study, we examined the role of ROS in Fas-mediated apoptosis and expression of cFLIP(L) and cFLIP(S). As expected, FasL treatment exhibited ROS-mediated apoptosis which was markedly blocked by the treatment of ROS scavengers NAC and GSH. The differential expression of cFLIP isoforms was also the result of post-transcriptional regulation, suggesting that cFLIP(S) expression is associated with the generation of ROS.

In conclusion, we revealed that FasL treatment caused downregulation of cFLIP(L) and upregulation of cFLIP(S). However, the upregulation of cFLIP(S) was not associated with apoptosis, instead the knockdown of cFLIP(S) eventually triggered FasL-mediated apoptosis. Furthermore, this differential expression of both variants was the result of post-transcriptional regulation and was highly associated with the generation of ROS in Caki cells. In the present study although we revealed the divergent expression of $\operatorname{cFLIP}(\mathrm{L}) /(\mathrm{S})$, there is still a strong need to explore the precise mechanism of the expression of cFLIP(S) during FasL-mediated cell death which may provide some insight to this complex apoptotic mechanism.

\section{Acknowledgements}

Not applicable.

\section{Funding}

The present study was supported by an NRF grant funded by the Korean Government (MSIP) (2014R1A5A2010008).

\section{Availability of data and materials}

The datasets used during the present study are available from the corresponding author upon reasonable request.

\section{Authors' contributions}

TKK conceived and designed the study. HJU and AKC performed the experiments. KJM and TKK wrote the manuscript. HJU, AKC, KJM and TKK reviewed and edited the manuscript. All authors read, approved the manuscript and agreed to be accountable for all aspects of the research in ensuring that the accuracy or integrity of any part of the work are appropriately investigated and resolved.

\section{Ethics approval and consent to participate}

Not applicable.

\section{Consent for publication}

Not applicable.

\section{Competing interests}

The authors declare that they have no competing interests.

\section{References}

1. Kikuchi H, Kuribayashi F and Imajoh-Ohmi S: Down-regulation of Fas-mediated apoptosis by plasma transglutaminase factor XIII that catalyzes fetal-specific cross-link of the Fas molecule. Biochem Biophys Res Commun 443: 13-17, 2014.

2. Wiegers GJ, Kaufmann M, Tischner D and Villunger A: Shaping the T-cell repertoire: A matter of life and death. Immunol Cell Biol 89: 33-39, 2011.

3. Kikuchi $\mathrm{H}$ and Nakayama T: GCN5 and BCR signalling collaborate to induce pre-mature $\mathrm{B}$ cell apoptosis through depletion of ICAD and IAP2 and activation of caspase activities. Gene 419: 48-55, 2008.

4. Geng L, Zhu B, Dai BH, Sui CJ, Xu F, Kan T, Shen WF and Yang JM: A let-7/Fas double-negative feedback loop regulates human colon carcinoma cells sensitivity to Fas-related apoptosis. Biochem Biophys Res Commun 408: 494-499, 2011.

5. Barnhart BC, Legembre P, Pietras E, Bubici C, Franzoso G and Peter ME: CD95 ligand induces motility and invasiveness of apoptosis-resistant tumor cells. EMBO J 23: 3175-3185, 2004

6. Wu XX, Mizutani Y, Kakehi Y, Yoshida O and Ogawa O: Enhancement of Fas-mediated apoptosis in renal cell carcinoma cells by adriamycin. Cancer Res 60: 2912-2918, 2000.

7. Arase H, Arase N and Saito T: Fas-mediated cytotoxicity by freshly isolated natural killer cells. J Exp Med 181: 1235-1238, 1995.

8. Itoh N, Yonehara S, Ishii A, Yonehara M, Mizushima S, Sameshima M, Hase A, Seto Y and Nagata S: The polypeptide encoded by the cDNA for human cell surface antigen Fas can mediate apoptosis. Cell 66: 233-243, 1991.

9. Kischkel FC, Hellbardt S, Behrmann I, Germer M, Pawlita M, Krammer PH and Peter ME: Cytotoxicity-dependent APO-1 (Fas/CD95)-associated proteins form a death-inducing signaling complex (DISC) with the receptor. EMBO J 14: 5579-5588, 1995.

10. Wang X, Wang Y, Lee SJ, Kim HP, Choi AM and Ryter SW: Carbon monoxide inhibits Fas activating antibody-induced apoptosis in endothelial cells. Med Gas Res 1: 8, 2011. 
11. Gloire G, Charlier E and Piette J: Regulation of CD95/APO-1/Fas-induced apoptosis by protein phosphatases. Biochem Pharmacol 76: 1451-1458, 2008.

12. Safa AR, Day TW and Wu CH: Cellular FLICE-like inhibitory protein (C-FLIP): A novel target for cancer therapy. Curr Cancer Drug Targets 8: 37-46, 2008.

13. Malhi $\mathrm{H}$ and Gores GJ: TRAIL resistance results in cancer progression: A TRAIL to perdition? Oncogene 25: 7333-7335, 2006.

14. Shin EC, Seong YR, Kim CH, Kim H, Ahn YS, Kim K, Kim SJ, Hong SS and Park JH: Human hepatocellular carcinoma cells resist to TRAIL-induced apoptosis, and the resistance is abolished by cisplatin. Exp Mol Med 34: 114-122, 2002.

15. Golks A, Brenner D, Fritsch C, Krammer PH and Lavrik IN c-FLIPR, a new regulator of death receptor-induced apoptosis. J Biol Chem 280: 14507-14513, 2005.

16. Sharp DA, Lawrence DA and Ashkenazi A: Selective knockdown of the long variant of cellular FLICE inhibitory protein augments death receptor-mediated caspase- 8 activation and apoptosis. J Biol Chem 280: 19401-19409, 2005.

17. Chang DW, Xing Z, Pan Y, Algeciras-Schimnich A Barnhart BC, Yaish-Ohad S, Peter ME and Yang X: c-FLIP(L) is a dual function regulator for caspase-8 activation and CD95-mediated apoptosis. EMBO J 21: 3704-3714, 2002.

18. Krueger A, Schmitz I, Baumann S, Krammer PH and Kirchhoff S: Cellular FLICE-inhibitory protein splice variants inhibit different steps of caspase-8 activation at the CD95 death-inducing signaling complex. J Biol Chem 276: 20633-20640, 2001.

19. Ram DR, Ilyukha V, Volkova T, Buzdin A, Tai A, Smirnova I and Poltorak A: Balance between short and long isoforms of cFLIP regulates Fas-mediated apoptosis in vivo. Proc Natl Acad Sci USA 113: 1606-1611, 2016.
20. Park YS, Kwon YJ and Chun YJ: CYP1B1 activates Wnt/ $\beta$-catenin signaling through suppression of Herc5-mediated ISGylation for protein degradation on $\beta$-catenin in HeLa cells. Toxicol Res 33: 211-218, 2017.

21. Jo Y and Shin DY: Repression of the F-box protein Skp2 is essential for actin damage-induced tetraploid G1 arrest. BMB Rep 50: 379-383, 2017.

22. Wang L, Azad N, Kongkaneramit L, Chen F, Lu Y, Jiang BH and Rojanasakul Y: The Fas death signaling pathway connecting reactive oxygen species generation and FLICE inhibitory protein down-regulation. J Immunol 180: 3072-3080, 2008.

23. Krueger A, Baumann S, Krammer PH and Kirchhoff S: FLICE-inhibitory proteins: Regulators of death receptor-mediated apoptosis. Mol Cell Biol 21: 8247-8254, 2001.

24. Thome M and Tschopp J: Regulation of lymphocyte proliferation and death by FLIP. Nat Rev Immunol 1: 50-58, 2001.

25. Medan D, Wang L, Toledo D, Lu B, Stehlik C, Jiang BH, Shi X and Rojanasakul Y: Regulation of Fas (CD95)-induced apoptotic and necrotic cell death by reactive oxygen species in macrophages. J Cell Physiol 203: 78-84, 2005.

26. Sato T, Machida T, Takahashi S, Iyama S, Sato Y, Kuribayashi K, Takada K, Oku T, Kawano Y, Okamoto T, et al: Fas-mediated apoptosome formation is dependent on reactive oxygen species derived from mitochondrial permeability transition in Jurkat cells. J Immunol 173: 285-296, 2004.

27. Vercammen D, Brouckaert G, Denecker G, Van de Craen M, Declercq W, Fiers W and Vandenabeele P: Dual signaling of the Fas receptor: Initiation of both apoptotic and necrotic cell death pathways. J Exp Med 188: 919-930, 1998. 